\title{
Oversampled Filter Banks
}

\author{
Zoran Cvetković, Member, IEEE, and Martin Vetterli, Fellow, IEEE
}

\begin{abstract}
Perfect reconstruction oversampled filter banks are equivalent to a particular class of frames in $\ell^{2}(Z)$. These frames are the subject of this paper. First, necessary and sufficient conditions on a filter bank for implementing a frame or a tight frame expansion are established, as well as a necessary and sufficient condition for perfect reconstruction using FIR filters after an FIR analysis. Complete parameterizations of oversampled filter banks satisfying these conditions are given. Further, we study the condition under which the frame dual to the frame associated with an FIR filter bank is also FIR and give a parameterization of a class of filter banks satisfying this property. Then, we focus on nonsubsampled filter banks. Nonsubsampled filter banks implement transforms similar to continuous-time transforms and allow for very flexible design. We investigate relations of these filter banks to continuous-time filtering and illustrate the design flexibility by giving a procedure for designing maximally flat two-channel filter banks that yield highly regular wavelets with a given number of vanishing moments.
\end{abstract}

\section{INTRODUCTION}

$\mathbf{T}$ THE IDEA OF localized time-frequency representations was introduced in the 1940's by Gabor [1], who proposed decompositions of signals in $L^{2}(\mathbf{R})$ in terms of modulated Gaussians, given that they attain the lower bound on the uncertainty in the joint time-frequency domain and thus facilitate signal analysis with good resolution in both time and frequency. Expansions based on different kinds of time-frequency localized waveforms have been subsequently used in physics, geophysics, and signal processing. However, only in the 1980's have they received a thorough and rigorous treatment. One of the important results was the discovery of the relationship between wavelet expansions in $L^{2}(\mathbf{R})$ and their discrete-time analog, which had been developing independently in the framework of filter banks and subband coding. Namely, wavelet bases in $L^{2}(\mathbf{R})$ can be generated from iterated filter banks [2], and these filter banks can be used for the efficient computation of corresponding continuous-time wavelet expansion coefficients [3]. The theory of time-frequency localized representations in $L^{2}(\mathbf{R})$ has been developed beyond linearly independent expansions, focusing on redundant expansions based on Weyl-Heisenberg and wavelet frames [4]. However, the theory of filter banks has been primarily confined to the critically sampled case,

Manuscript received December 30, 1994; revised April 18, 1997. This work was supported in part by the National Science Foundation under Grant MIP90-14189. This work was performed while the authors were with the Electrical Engineering and Computer Science Department, University of California at Berkeley. The associate editor coordinating the review of this paper and approving it for publication was Prof. Roberto H. Bamberger.

Z. Cvetković is with AT\&T Laboratories-Research, Florham Park, NJ 07932 USA.

M. Vetterli is with EPF, Lausanne (EPFL), Lausanne, Switzerland and with the Electrical Engineering and Computer Science Department, University of California, Berkeley, CA 94720 USA.

Publisher Item Identifier S 1053-587X(98)03301-7. i.e., orthonormal and biorthonormal bases in $\ell^{2}(\mathbf{Z})$ [5], [6]. In this paper, we study the class of frames in $\ell^{2}(\mathbf{Z})$, which are equivalent to perfect reconstruction oversampled filter banks.

One of the main reasons for studying overcomplete expansions is that the requirement for orthogonality or linear independence of expansion vectors imposes considerable constraints that can be in conflict with other design specifications. Perhaps the most striking example is the fact that Gabor analysis with good time-frequency resolution is not possible with orthonormal bases [7], [8]. For a number of applications, orthogonality is indeed not needed. For instance, it is hard to believe that orthonormal representations occur anywhere in nature and that they would be appropriate for modeling biological systems or natural phenomena. Redundant representations followed by a sophisticated selection of information can even yield good compression schemes. This has been demonstrated by Mallat and Zhong with their wavelet modulus maxima signal compression algorithm [9]. The matching pursuit algorithm, studied also by Mallat et al. [10], is another example. It is based on the idea that there is a greater chance for finding compact signal representations if the dictionary of vectors at our disposal is richer. The full potential of these algorithms has not been assessed yet; however, they already give results comparable with standard compression schemes [11].

The issues on overcomplete expansions that we investigate here are related to the completeness and the stability of subband decompositions provided by oversampled filter banks. In particular, we investigate

- the necessary and sufficient conditions on filter banks for implementing frame or tight frame expansions;

- the feasibility of perfect reconstruction using FIR filters after an FIR analysis;

- the design of oversampled filter banks;

- the relation to continuous-time signal analysis.

General results regarding oversampled filter banks, such as the frame and the tight frame conditions, are given in Section III. Section III also provides complete parameterizations of FIR filter bank frames and tight frames. Parahermitan unimodular matrices of polynomials give FIR filter bank frames whose dual frames also consist of finite length vectors. A complete parameterization of these matrices is also given in Section III. The frame and tight frame conditions on the nonsubsampled filter banks, as well as the conditions for FIR reconstruction, have special forms, and these are studied in Section IV. In the same section, we discuss a design of highly regular wavelets with a given number of vanishing moments that are obtained from the iterated octave band structure.

Notation: For a filter $H(z), \tilde{H}(z)$ will denote the filter whose impulse response is the complex conjugate of the timereversed version of the impulse response of $H(z)$. Similarly, 
when used with matrices of rational functions of the complex variable $z, \tilde{\mathbf{H}}(z)$ will denote the matrix obtained from $\mathbf{H}(z)$ by transposing it, conjugating all of the coefficients of the rational functions in $\mathbf{H}(z)$, and replacing $z$ by $z^{-1}$. If $\tilde{\mathbf{H}}(z)=\mathbf{H}(z)$, we say that $\mathbf{H}(z)$ is parahermitian. A polynomial matrix $\mathbf{H}(z)$, whose determinant is a nonzero constant, is called a unimodular matrix. A matrix $\mathbf{H}(z)$ is said to be paraunitary if $\tilde{\mathbf{H}}(z) \mathbf{H}(z)=c \mathbf{I}$, where $\mathbf{I}$ is the identity matrix, and $c$ is a constant. Note that in this paper we will use the term polynomial for Laurent polynomials, in general, that is, FIR filters $H(z)=\sum_{i=n}^{m} h_{i} z^{i}$ that contain possibly both positive and negative powers of $z$. The complex conjugate transpose of a vector $\mathbf{v}$ will be denoted as $\mathbf{v}^{*}$; when used with scalars, the $*$ superscript will denote complex conjugation.

\section{Filter BANKS AND Frame ExPANSIONS}

The theory of filter banks [5], [6] provides a convenient framework for both the study and the implementation of an important class of signal decompositions in $\ell^{2}(\mathbf{Z})$. These are expansions underlying signal analysis through a sliding window using a selected set of elementary waveforms. In general, they have the form

$$
x[n]=\sum_{i=0}^{K-1} \sum_{j=-\infty}^{\infty} c_{i, j} \varphi_{i, j}[n]
$$

where the vectors $\varphi_{i, j}[n]$ denote the translated versions of $K$ elementary waveforms

$$
\varphi_{i, j}[n]=\varphi_{i}[n-j N]
$$

and $N \leq K$. Any signal in $\ell^{2}(\mathbf{Z})$ can be represented in a numerically stable way using such an expansion if and only if the family $\Phi$

$$
\begin{array}{r}
\Phi=\left\{\varphi_{i, j}: \varphi_{i, j}[n]=\varphi_{i}[n-j N]\right. \\
i=0,1, \ldots, K-1, j \in \mathbf{Z}\}
\end{array}
$$

constitutes a frame in $\ell^{2}(\mathbf{Z})$. Here, we briefly review results of the frame theory that are relevant for this paper. This review is based on the treatments given in [2] and [12].

A family of vectors $\Phi$ given by (2) is said to be a frame if for any $x \in \ell^{2}(\mathbf{Z})$

$$
A\|x\|^{2} \leq \sum_{i=0}^{K-1} \sum_{j=-\infty}^{\infty}\left|\left\langle x, \varphi_{i, j}\right\rangle\right|^{2} \leq B\|x\|^{2}
$$

for some constants $A>0$ and $B<\infty$, which are called frame bounds. If the family $\Phi$ is a frame, there exists another frame

$$
\begin{array}{r}
\Psi=\left\{\psi_{i, j}: \psi_{i, j}[n]=\psi_{i}[n-j N]\right. \\
i=0,1, \ldots, K-1, j \in \mathbf{Z}\}
\end{array}
$$

such that the coefficients of the expansion in (1) can be calculated as inner products with its vectors, that is

$$
x[n]=\sum_{i=0}^{K-1} \sum_{j=-\infty}^{\infty}\left\langle x, \psi_{i, j}\right\rangle \varphi_{i, j}[n] .
$$

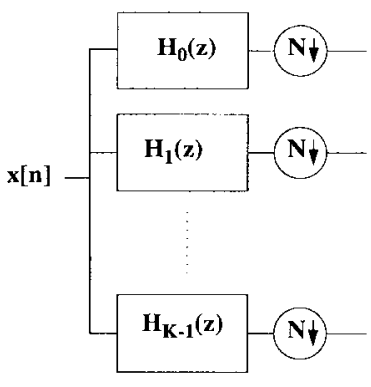

(a)

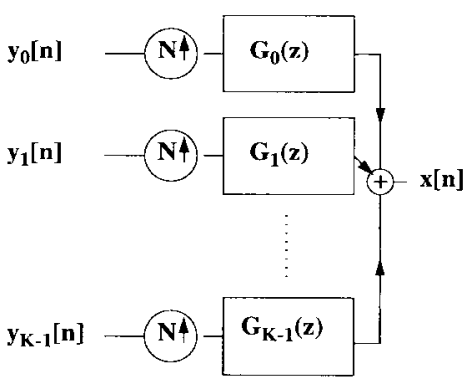

(b)
Fig. 1. $K$-channel filter bank with subsampling by factor $N$ in the channels. (a) Analysis filter bank. (b) Synthesis filter bank.

Frames $\Phi$ and $\Psi$ have interchangeable roles so that any signal in $\ell^{2}(\mathbf{Z})$ can also be expanded as

$$
x[n]=\sum_{i=0}^{K-1} \sum_{j=-\infty}^{\infty}\left\langle x, \varphi_{i, j}\right\rangle \psi_{i, j}[n] .
$$

For a given analysis frame $\Phi$, the corresponding synthesis frame $\Psi$ in (6) is not unique. One particular solution is the frame dual to $\Phi$ [12]. When a signal is synthesized from expansion coefficients degraded by an additive noise, the noise component that is orthogonal to the range of the frame expansion, is projected to zero, provided that the synthesis is performed using the dual frame. No other synthesis frame has this maximal noise reduction property.

If the frame bounds are equal $(A=B)$, we say that the frame is tight. It can be shown that under this condition, the frame $\Phi$ is equal to its dual. Hence, the expansion formula (1) has a form similar to orthogonal expansions

$$
x[n]=\frac{1}{A} \sum_{i=0}^{K-1} \sum_{j=-\infty}^{\infty}\left\langle x, \varphi_{i, j}\right\rangle \varphi_{i, j}[n] .
$$

However, unlike an orthogonal basis, vectors of a tight frame are generally not linearly independent.

The inner products of a signal $x$ with the vectors of the family $\Phi,\left\langle x, \varphi_{i, j}\right\rangle$ can be obtained at the output of a $K$ channel filter bank with subsampling by factor $N$ in each of the channels [see Fig. 1(a)]. The impulse responses of the filters $H_{0}(z), H_{1}(z), \ldots, H_{K-1}(z)$ of such a filter bank are the complex conjugates of the time-reversed versions of the elementary waveforms $\varphi_{i}, h_{i}[n]=\varphi_{i}^{*}[-n], i=0,1, \ldots, K-$ 1. If $\Phi$ is a frame, the filter bank associated with $\Phi$ in this way is said to implement a frame expansion, and $\Phi$ is called its associated frame or a filter bank frame. Note that $\Phi$ can be a frame only if $N \leq K$, and we assume this condition throughout this paper. Critically sampled filter banks are those where $N=K$, whereas the term oversampled filter banks refers to the case $N<K$. In filter bank terms, $\Phi$ being a frame means that any signal can be reconstructed in a numerically stable $^{1}$ manner from the subband components obtained at the outputs of the associated filter bank. This reconstruction is implemented using a synthesis filter bank such as the one

\footnotetext{
${ }^{1}$ Stable reconstruction means that a bounded perturbation of subband components cannot result in an arbitrary large error and that a small perturbation can cause only a small reconstruction error.
} 
shown in Fig. 1(b). If the impulse responses of the filters $G_{0}(z), \ldots, G_{K-1}(z)$ of the synthesis filter bank are equal to the waveforms $\psi_{i}$, that is, $g_{i}[n]=\psi_{i}[n]$, then this filter bank implements the synthesis formula

$$
x[n]=\sum_{i=0}^{K-1} \sum_{j=-\infty}^{\infty} y_{i}[n-k] \psi_{i, j}[k]
$$

where $y_{i}[n]$ denotes the sequence at the input of the $i$ th channel.

The scope of frames in $\ell^{2}(\mathbf{Z})$ that can be derived from filter banks goes beyond families of vectors of the type given in (2). An abundance of frames can be generated by iteratively growing filter bank trees in the manner used for generating wavelet packets [16]. The significance of iterated filter structures is that they can produce waveforms with almost any reasonable localization in the time-frequency plane, which allows for signal analysis with a great flexibility of time and frequency resolutions.

\section{General Results on Oversampled Filter BANKS}

\section{A. Frame Conditions}

The frame conditions on an oversampled filter bank will be expressed here in terms of properties of its polyphase analysis matrix. In the case of a $K$-channel filter bank

$$
\left\{H_{0}(z), H_{1}(z), \ldots, H_{K-1}(z)\right\}
$$

and a subsampling factor $N$ [see Fig. 1(a)], the polyphase analysis matrix $\mathbf{H}(z)$ is defined as

$$
\mathbf{H}(z)=\left[\begin{array}{ccc}
H_{00}(z) & \cdots & H_{0(N-1)}(z) \\
H_{10}(z) & \cdots & H_{1(N-1)}(z) \\
\cdots & \cdots & \cdots \\
H_{(K-1) 0}(z) & \cdots & H_{(K-1)(N-1)}(z)
\end{array}\right]
$$

where

$$
H_{i j}(z)=\sum_{n=-\infty}^{+\infty} h_{i}[n N-j] \cdot z^{-n}
$$

represents the $j$ th polyphase component of $H_{i}(z)$. The analysis filters are given in terms of their polyphase components by

$$
H_{i}(z)=\sum_{j=0}^{N-1} z^{j} \cdot H_{i j}\left(z^{N}\right) .
$$

The necessary and sufficient frame and tight frame conditions are given by the following two theorems, which are proven in Appendices A and B, respectively.

Theorem 1: A filter bank implements a frame expansion if and only if its polyphase analysis matrix is of full rank on the unit circle.
Theorem 2: A filter bank implements a tight frame expansion if and only if its polyphase analysis matrix is paraunitary $\tilde{\mathbf{H}}(z) \mathbf{H}(z)=c \mathbf{I}$.

In this paper, we are primarily interested in FIR filter banks. However, note that Theorems 1 and 2 are also valid for IIR filter banks if we assume stable filters (not necessarily causal). The proofs go along the same lines as in the FIR case.

Another formulation of the frame condition is given by the following corollary of Theorem 1 . The proof of this corollary is straightforward and will not be given here.

Corollary 1: A filter bank with the polyphase analysis matrix $\mathbf{H}(z)$ implements a frame expansion if and only if there exists a matrix $\mathbf{G}(z)$ of stable rational functions, ${ }^{2}$ which is a left inverse of $\mathbf{H}(z)$

$$
\mathbf{G}(z) \mathbf{H}(z)=c \mathbf{I}
$$

One convenience of this formulation is that it explicitly gives a synthesis frame corresponding to the frame associated with the analysis filter bank. Namely, the left inverse

$$
\mathbf{G}(z)=\left[\begin{array}{ccc}
G_{00}(z) & \cdots & G_{(K-1) 0}(z) \\
G_{01}(z) & \cdots & G_{(K-1) 1}(z) \\
\cdots & \cdots & \cdots \\
G_{0(N-1)}(z) & \cdots & G_{(K-1)(N-1)}(z)
\end{array}\right]
$$

in (12) is the so-called synthesis polyphase matrix. Its entries are the polyphase components of filters of a synthesis filter bank [Fig. 1(b)], which can be used for perfect reconstruction of a signal from the decomposition obtained at the output of the analysis filter bank. The synthesis filters are given in terms of entries of $\mathbf{G}(z)$ as

$$
G_{i}(z)=\sum_{j=0}^{N-1} z^{-j} G_{i j}\left(z^{N}\right)
$$

[note the inversion of the powers of $z$ with respect to (11)].

If the frame condition of Theorem 1 is satisfied, the solution for $\mathbf{G}(z)$ of the polyphase equation (12), and, hence, the synthesis filter bank, is not unique in the oversampled case $(N<K)$. This is in accordance with the fact that for a given analysis frame, the corresponding synthesis frame is not unique. One solution for $\mathbf{G}(z)$ is the parapseudoinverse of $\mathbf{H}(z)$, which is given by

$$
\mathbf{H}^{+}(z)=(\tilde{\mathbf{H}}(z) \mathbf{H}(z))^{-1} \tilde{\mathbf{H}}(z)
$$

It can be easily verified that the frame corresponding to $\mathbf{G}(z)=\mathbf{H}^{+}(z)$ is the frame dual to the frame associated with the analysis filter bank. The following theorem gives a necessary and sufficient condition for an FIR filter bank frame to have the dual consisting of finite length filters. It is proven in Appendix C.

Theorem 3: For a frame associated with an FIR filter bank with the polyphase analysis matrix $\mathbf{H}(z)$, its dual frame consists of finite length vectors if and only if $\tilde{\mathbf{H}}(z) \mathbf{H}(z)$ is unimodular.

Note that in the oversampled case, Theorem 3 does not preclude the existence of an FIR synthesis filter bank even if

\footnotetext{
${ }^{2}$ These functions need not be causal.
} 
$\tilde{\mathbf{H}}(z) \mathbf{H}(z)$ is not unimodular. However, due to the aforementioned reasons, we put the emphasis on reconstruction using dual frames.

In the critically sampled case $(K=N)$, the situation is different. The frame associated with a perfect reconstruction critically sampled filter bank is a Riesz bases [6] in $\ell^{2}(\mathbf{Z})$. The corresponding synthesis frame, i.e., the left inverse of $\mathbf{H}(z)$, is unique, and it is the dual of the analysis frame. The following result on critically sampled filter banks can be established as a corollary of Theorem 3 .

Corollary 2 [6]: Perfect reconstruction with FIR filters after an analysis by a critically sampled FIR filter bank is possible if and only if the determinant of $\mathbf{H}(z)$ is a pure delay.

\section{B. Parameterization of Frames in $\ell^{2}(\mathbf{Z})$}

The parameterization of filter bank frames given here is based on the Smith form of polynomial matrices [13]. A polynomial matrix $\mathbf{H}(z)$ of dimension $K \times N(K \geq N)$ can be decomposed as the product

$$
\mathbf{H}(z)=\mathbf{R}(z) \mathbf{D}(z) \mathbf{C}(z)
$$

where $\mathbf{R}(z)$ and $\mathbf{C}(z)$ are unimodular matrices of dimensions $K \times K$ and $N \times N$, respectively, and $\mathbf{D}(z)$ is a diagonal $K \times N$ polynomial matrix

$$
\mathrm{D}(z)=\left[\begin{array}{cccc}
d_{1}(z) & 0 & \ldots & 0 \\
0 & d_{2}(z) & \ldots & 0 \\
\ldots & \ldots & \ldots & \ldots \\
0 & 0 & \ldots & d_{N}(z) \\
0 & 0 & \ldots & 0 \\
\ldots & \ldots & \ldots & \ldots \\
0 & 0 & \ldots & 0
\end{array}\right] .
$$

The unimodular matrices can be chosen so that the polynomials $d_{i}(z)$ are monic, and $d_{i}(z)$ is a factor of $d_{i+1}(z)$. Such a matrix $\mathbf{D}(z)$ is called the Smith form of $\mathbf{H}(z)$. The unimodular matrices $\mathbf{R}(z)$ and $\mathbf{C}(z)$ are products of finitely many elementary matrices

$$
\begin{aligned}
& \mathbf{R}(z)=\mathbf{R}_{1}(z) \mathbf{R}_{2}(z) \cdots \mathbf{R}_{m}(z) \\
& \mathbf{C}(z)=\mathbf{C}_{1}(z) \mathbf{C}_{2}(z) \cdots \mathbf{C}_{n}(z) .
\end{aligned}
$$

The elementary matrices $\mathbf{R}_{i}(z)$ and $\mathbf{C}_{j}(z)$ correspond to elementary row and column operations, respectively, and have one of the following forms:

- a permutation matrix, i.e., the identity matrix with permuted rows;

- a diagonal matrix with elements on the diagonal equal to unity, except for one that is equal to a nonzero constant;

- a matrix with ones on the main diagonal and a single nonzero entry off the diagonal, which is a polynomial $\alpha(z)$.

An example of the three types of $4 \times 4$ elementary matrices is given as follows.

$$
\left[\begin{array}{llll}
1 & 0 & 0 & 0 \\
0 & 0 & 0 & 1 \\
0 & 0 & 1 & 0 \\
0 & 1 & 0 & 0
\end{array}\right],\left[\begin{array}{llll}
1 & 0 & 0 & 0 \\
0 & 1 & 0 & 0 \\
0 & 0 & c & 0 \\
0 & 0 & 0 & 1
\end{array}\right],\left[\begin{array}{cccc}
1 & 0 & 0 & 0 \\
0 & 1 & 0 & 0 \\
\alpha(z) & 0 & 1 & 0 \\
0 & 0 & 0 & 1
\end{array}\right] .
$$

A complete parameterization of FIR filter bank frames follows directly from the Smith form and is given by the next proposition.

Proposition 1: An FIR filter bank implements a frame expansion if and only if the polynomials on the diagonal of the Smith form of its polyphase analysis matrix have no zeros on the unit circle.

This proposition follows from the fact that the polyphase matrix and its Smith form have the same rank on the unit circle since they are related by elementary row (column) operations. Hence, the filter bank implements a frame decomposition if and only if its Smith form is of full rank on the unit circle and that holds if and only if the polynomials on the diagonal have no zeros on the unit circle.

An important class of FIR filter bank frames are those that have duals consisting of finite length vectors. According to Theorem 3 , these are equivalent to polynomial matrices $\mathbf{H}(z)$ such that $\tilde{\mathbf{H}}(z) \mathbf{H}(z)$ is unimodular and positive definite on the unit circle. A parameterization of these frames is given by the following proposition.

Proposition 2: Consider an oversampled FIR filter bank with the polyphase analysis matrix $\mathbf{H}(z)$. $\tilde{\mathbf{H}}(z) \mathbf{H}(z)$ is unimodular and positive definite on the unit circle if $\mathbf{H}(z)$ can be written as

$$
\mathbf{H}(z)=\mathbf{H}_{0} \mathbf{R}(z) \mathbf{D}(z) \mathbf{C}(z)
$$

where the factors $\mathbf{H}_{0}, \mathbf{R}(z), \mathbf{D}(z)$, and $\mathbf{C}(z)$ have the following forms.

- $\mathbf{H}_{0}$ is a $K \times N$ matrix of scalars, such that $\tilde{\mathbf{H}}_{0} \mathbf{H}_{0}=c \mathbf{I}$.

- $\mathbf{R}(z)$ and $\mathbf{C}(z)$ are $N \times N$ unimodular matrices, which are products of finitely many elementary matrices.

- $\mathrm{D}(z)$ is an $N \times N$ diagonal matrix of nonzero monomials.

On the other hand, any unimodular parahermitian matrix of polynomials $\mathbf{P}(z)$, which is positive definite on the unit circle, can be factored as $\mathbf{P}(z)=\tilde{\mathbf{H}}(z) \mathbf{H}(z)$, where $\mathbf{H}(z)$ is of the form given in (18).

This result is proven in Appendix D.

For perfect reconstruction using an FIR filter bank after analysis with an FIR oversampled filter bank, $\tilde{\mathbf{H}}(z) \mathbf{H}(z)$ need not be unimodular. A necessary and sufficient condition for the feasibility of an FIR synthesis is given by the following proposition. It is proven in Appendix E.

Proposition 3: Perfect reconstruction with FIR filters after analysis by an FIR filter bank is possible if and only if the polynomials on the diagonal of the Smith form of the polyphase analysis matrix are monomials.

As it was shown in the previous subsection, tight filter bank frames are equivalent to paraunitary polynomial matrices. A $K \times N$ paraunitary matrix $(K>N)$ can always be embedded into a $K \times K$ paraunitary matrix [15]. The following parameterization of rectangular paraunitary polyphase matrices, that is, tight filter bank frames, follows directly from one of the factorizations of square paraunitary matrices studied by Vaidyanathan [5].

Proposition 4: A $K \times N(K>N)$ polynomial matrix $\mathbf{H}(z)$ is paraunitary if and only if it has the decomposition

$$
\mathbf{H}(z)=\mathbf{V}_{M}(z) \mathbf{V}_{M-1}(z) \cdots \mathbf{V}_{1}(z) \mathbf{H}_{0} .
$$




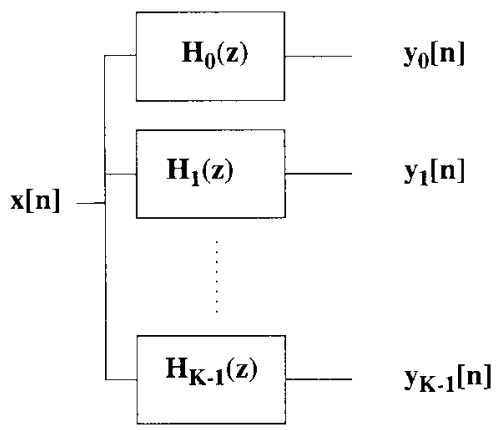

(a)

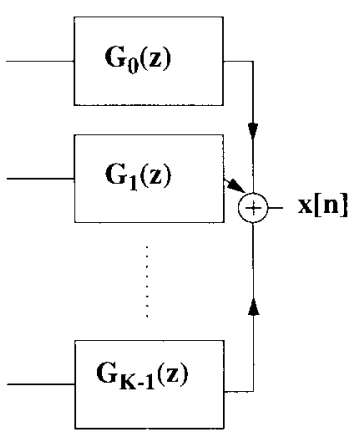

(b)
Fig. 2. $\quad K$-channel nonsubsampled filter bank. (a) Analysis filter bank. (b) Synthesis filter bank.

The elementary building blocks $\mathbf{V}_{i}(z)$ have the form

$$
\mathbf{V}_{i}(z)=\mathbf{I}-\mathbf{v}_{i} \mathbf{v}_{i}^{*}+z^{-1} \mathbf{v}_{i} \mathbf{v}_{i}^{*}
$$

where $\mathbf{v}_{\boldsymbol{i}}$ denotes a unit norm vector, and $\mathbf{H}_{0}$ is a $K \times N$ matrix of scalars such that $\tilde{\mathbf{H}}_{0} \mathbf{H}_{0}=c \mathbf{I}$.

\section{NONSUBSAMPLED FILTER BANKS}

Nonsubsampled filter banks have several nice properties. Constraints for perfect reconstruction are mild and allow for very flexible design. The highly redundant representations they generate can be close discrete-time approximations of continuous-time transforms. Examples of applications for which that property is crucial are applications based on wavelet modulus maxima representations, which are proposed by Mallat et al. [18] for singularity detection, signal denoising, and compression, and which use nonsubsampled filter banks as the preprocessing tool. Nonsubsampled filter banks also find their place in applications that require shift-invariant representations, a requirement that conflicts with subsampling in the filter bank channels [17].

\section{A. General Results on Nonsubsampled Filter Banks}

In this subsection, we discuss the frame conditions for the case of nonsubsampled filter banks, as well as the condition for the feasibility of an FIR synthesis after an FIR analysis.

The polyphase analysis matrix of a nonsubsampled filter bank [see Fig. 2(a)] is a column vector whose entries are the analysis filters themselves

$$
\mathbf{H}(z)=\left[\begin{array}{llll}
H_{0}(z) & H_{1}(z) & \cdots & H_{K-1}(z)
\end{array}\right]^{T} .
$$

Perfect and stable reconstruction is possible, provided that there exist stable filters $G_{0}(z), G_{1}(z), \ldots, G_{K-1}(z)$ such that

$$
H_{0}(z) G_{0}(z)+H_{1}(z) G_{1}(z)+\cdots+H_{K-1}(z) G_{K-1}(z)=1 \text {. }
$$

In that case, reconstruction is performed by a synthesis filter bank with the filters $G_{i}(z)$ [see Fig. 2(b)]. The necessary and sufficient condition for the existence of such filters is given by the following corollary of Theorem 1.
Corollary 3: A nonsubsampled filter bank implements a frame decomposition if and if only its analysis filters have no zeros in common on the unit circle.

The frame condition does not guarantee that FIR reconstruction is possible.

Corollary 4: Perfect reconstruction using FIR filters after an FIR analysis by a nonsubsampled filter bank is possible if and only if the analysis filters have no zeros in common.

This result is a corollary of Proposition 3. As a special case of Theorem 2, we have the following result about nonsubsampled filter banks and tight frames.

Corollary 5: A nonsubsampled filter bank implements a tight frame expansion if and only if its analysis filters are power complementary.

$$
H_{0}(z) \tilde{H}_{0}(z)+H_{1}(z) \tilde{H}_{1}(z)+\cdots+H_{K-1}(z) \tilde{H}_{K-1}(z)=c .
$$

In the case of nonsubsampled filter banks, only if a frame associated with an FIR filter bank is tight does its dual frame consist of finite length vectors. This result is an immediate corollary of Theorem 3 .

Corollary 6: For a frame associated with an FIR nonsubsampled filter bank, its dual frame consists of finite length vectors if and only if the analysis filters are power complementary, that is, if and only if the frame is tight.

\section{B. Nonsubsampled Filter Banks and Continuous Time Signal Analysis}

A nonsubsampled filter bank, as shown in the following, gives samples of continuous-time transforms of a signal in $L^{2}(\mathbf{R})$, provided that an appropriate discrete-time version of the signal is available at the input. In this subsection, we identify the underlying continuous-time filters. An important particular case is that of two-channel octave band iterated filter banks that yield samples of the continuous wavelet transform.

The discretization of a continuous-time signal in $L^{2}(\mathbf{R})$ usually amounts to projecting it onto an approximation space $V_{\varphi}$, which is spanned by translates of a single function $\varphi(x)$ called the generating function. The generating function should satisfy a condition ${ }^{3}$

$$
0<\alpha \leq \sum_{k \in \mathbf{Z}}|\hat{\varphi}(\omega+2 k \pi)|^{2} \leq \beta<\infty
$$

which means that its translates by integer shifts constitute a Riesz basis for $V_{\varphi}$. The projection $f_{p}(x)$ of a signal $f(x)$ onto $V_{\varphi}$ can be represented by a sequence $f_{\phi}[n]$ that consists of coefficients of the expansion

$$
f_{p}(x)=\sum_{n \in \mathbf{Z}} f_{\phi}[n] \varphi(x-n) .
$$

The sequence $f_{\phi}[n]$ is a discrete-time version of the signal and is obtained by sampling $f(x)$ prefiltered by an appropriate filter $\phi(x)$

$$
f_{\phi}[n]=\int_{-\infty}^{+\infty} f(x) \phi(n-x) d x .
$$

\footnotetext{
${ }^{3}$ In this paper, the Fourier transform of a signal $f(x)$ will be written as $\hat{f}(\omega)$.
} 


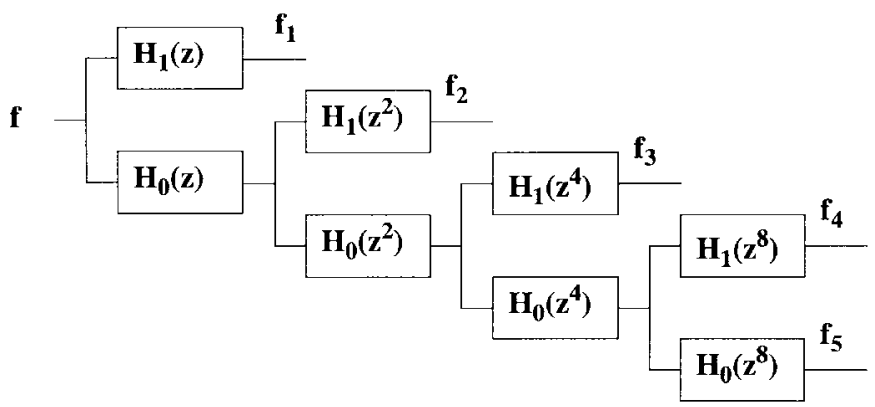

Fig. 3. Octave band iterated filter bank for discrete-time wavelet transform of depth $J=4$.

Integer translates of the time-reversed version of $\phi(x)$ constitute another basis for $V_{\varphi}$, which is dual to $\{\varphi(x-n), n \in \mathbf{Z}\}$ [19]. The projection $f_{p}(x)$ can be alternatively represented as

$$
f_{p}(x)=\sum_{n \in \mathbf{Z}} f_{\varphi}[n] \phi(n-x)
$$

where

$$
f_{\varphi}[n]=\int_{-\infty}^{+\infty} f(x) \varphi(x-n) d x .
$$

Shannon's sampling occurs when the approximation space is the space of bandlimited signals generated by translates of the sinc function. Another particular case is the $V_{0}$ space of Mallat's multiresolution analysis, with $\varphi(x)$ being the corresponding scaling function. For a detailed analysis of various aspects of generalized sampling, refer to the work of Unser and Aldroubi [19].

For a continuous-time signal $f(x)$ discretized as in (26), consider a subsequent processing by the nonsubsampled filter bank $\left\{H_{0}(z), H_{1}(z), \ldots, H_{K-1}(z)\right\}$. The subband components $f_{k}, k=0,1,2, \ldots K-1$ generated by the filter bank are given in the Fourier domain by

$$
\hat{f}_{k}\left(e^{j \omega}\right)=H_{k}\left(e^{j \omega}\right) \sum_{n \in \mathbf{Z}} \hat{f}(\omega+2 n \pi) \hat{\phi}(\omega+2 n \pi) .
$$

This expression indicates that the filter bank performs regular sampling of signals obtained by filtering $f(x)$ by a set of filters $\varphi_{0}(x), \varphi_{1}(x), \ldots, \varphi_{K-1}(x)$. These filters are given in the Fourier domain as

$$
\hat{\varphi}_{k}(\omega)=H_{k}\left(e^{j \omega}\right) \hat{\phi}(\omega) .
$$

This characterizes the continuous-time transforms that underly the nonsubsampled filter bank analysis in the given approximation space $V_{\varphi}$.

An interesting particular case is wavelet analysis implemented using an iterated two-channel filter bank, such as the one shown in Fig. 3. Suppose that the lowpass filter $H_{0}(z)$ of this filter bank is regular, i.e., that the infinite product

$$
\lim _{n \rightarrow \infty} \prod_{s=1}^{n} H_{0}\left(e^{j 2^{-s} \omega}\right)
$$

converges to $\hat{\phi}(\omega)$, which is the Fourier transform of a continuous-time function $\phi(x) \in L^{2}(\mathbf{R})$. If this particular $\phi(x)$ is used in the discretization given by (26), then the

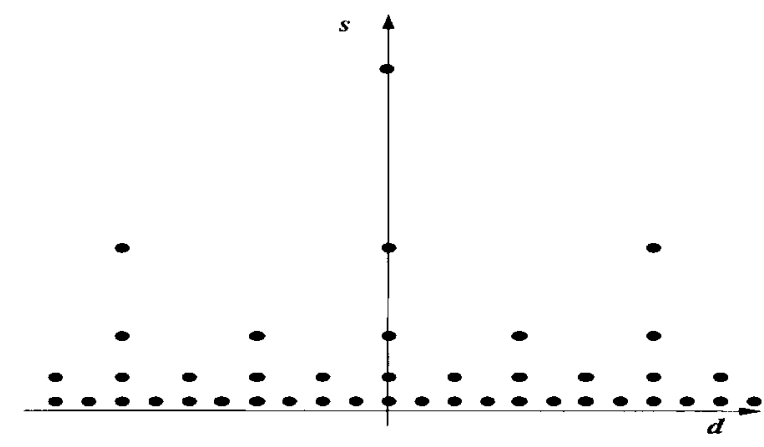

(a)

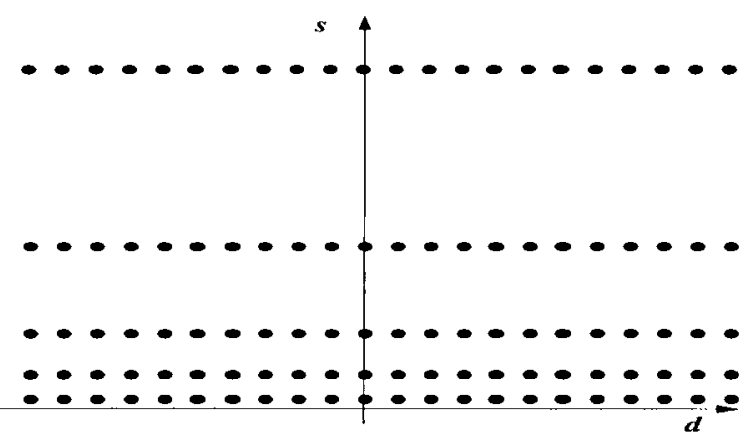

(b)

Fig. 4. Sampling grids of the continuous wavelet transform implemented by discrete-time processing. (a) Grid corresponding to the two-channel critically sampled filter bank. (b) Grid corresponding to the two-channel nonsubsampled filter bank.

filter bank yields samples, up to a multiplicative factor, of the continuous wavelet transform of $f(x)$. The continuous wavelet transform of $f(x)$ for a wavelet $\psi(x)$ is defined as

$$
C W T_{f}(a, b)=\int_{-\infty}^{\infty} \psi_{a, b}^{*}(x) f(x) d x
$$

where $\psi_{a, b}(x)=\frac{1}{\sqrt{a}} \psi\left(\frac{x-b}{a}\right)$. The sequence $f_{k}[n]$, which is obtained in the $k$ th channel (see Fig. 3), is the sequence of samples

$$
f_{k}[n]=\int_{-\infty}^{\infty} f(x) \frac{1}{2^{k}} \psi\left(\frac{n-x}{2^{k}}\right) d x
$$

where $\psi(x)$ is the wavelet given by

$$
\hat{\psi}(\omega)=H_{1}\left(e^{\frac{j \omega}{2}}\right) \hat{\phi}\left(\frac{\omega}{2}\right) .
$$

Therefore, the octave band iterated filter bank of depth $J$ provides the samples of the continuous wavelet transform ${ }^{4}$ at the grid given by

$$
(a, b) \in\left\{\left(2^{i}, j\right): i=1,2, \ldots, J \quad j \in \mathbf{Z}\right\} .
$$

Besides sampling the continuous wavelet transform on a denser grid as compared with a critically sampled filter bank (see Fig. 4), a nonsubsampled filter bank allows for a more flexible design. This flexibility is demonstrated in the next subsection.

\footnotetext{
${ }^{4}$ We assume that the wavelet $\psi(x)$ is real.
} 
TABLE I

Autocorrelation Functions of the LowPass Filters $H_{0}(z)$ FOR POWER Complementary Nonsubsampled Filter Banks $\left(H_{0}(z), H_{1}(z)\right)$. The Autocorrelation Functions Are Given for Several Filter Lengths $L$ AND With DifFerent Multiplicities $N_{1}$ OF THE Zero at $z=1$ OF THE Highpass Filters $H_{1}(z)$. The Autoccorelation Functions Are Symmtric So Only the First $L$ Out of $2 L-1$ Coefficients Are Given in the Table

\begin{tabular}{r|r|r}
\hline$L$ & $N_{1}$ & $H_{0}(z) H_{0}\left(z^{-1}\right)$ \\
\hline 4 & 1 & $\frac{1}{2^{6}}[1,6,15,20]$ \\
4 & 2 & $\frac{1}{2^{5}}[-1,0,9,16]$ \\
5 & 1 & $\frac{1}{2^{8}}[1,8,28,56,70]$ \\
5 & 2 & $\frac{1}{2^{8}}[-3,-8,12,72,110]$ \\
6 & 1 & $\frac{1}{2^{10}}[1,10,45,120,210,252]$ \\
6 & 2 & $\frac{1}{2^{8}}[-1,-5,-5,20,70,98]$ \\
6 & 3 & $\frac{1}{2^{9}}[3,0,-25,0,150,256]$ \\
7 & 1 & $\frac{1}{2^{12}}[1,12,66,220,495,792,924]$ \\
7 & 2 & $\frac{1}{2^{12}}[-5,-36,-90,-20,405,1080,1428]$ \\
7 & 3 & $\frac{1}{2^{13}}[5,12,-30,-100,75,600,924]$ \\
8 & 1 & $\frac{1}{2^{14}}[1,4,91,364,1001,2002,3003,3432]$ \\
8 & 2 & $\frac{1}{2^{13}}[-3,-28,-105,-168,77,924,2079,2640]$ \\
8 & 3 & $\frac{1}{2^{14}}[15,70,21,-420,-665,1050,4725,6792]$ \\
8 & 4 & $\frac{1}{2^{12}}[-5,0,49,0,-245,0,1225,2048]$ \\
9 & 1 & $\frac{1}{2^{16}}[1,16,120,560,1820,4368,8008,11440,12870]$ \\
9 & 2 & $\frac{1}{2^{16}}[-7,-80,-392,-1008,-1092,1456,8008,16016,19734]$ \\
9 & 3 & $\frac{1}{2^{16}}[21,144,280,-336,-2100,-1904,5544,18480,25278]$ \\
9 & 4 & $\frac{1}{2^{16}}[-35,-80,280,784,-980,-3920,1960,19600,30318]$ \\
10 & 1 & $\frac{1}{2^{18}}[1,18,153,816,3060,8568,18564,31824,43758,48620]$ \\
10 & 2 & $\frac{1}{2^{16}}[-2,-27,-162,-552,-1080,-756,2184,8424,15444,18590]$ \\
10 & 3 & $\frac{1}{2^{16}}[7,63,207,168,-756,-2268,-1092,6552,18018,23738]$ \\
10 & 4 & $\frac{1}{2^{16}}[-14,-63,18,504,504,-1764,-3528,3528,19404,28358]$ \\
10 & 5 & $\frac{1}{2^{17}}[35,0,-405,0,2268,0,-8820,0,39690,65536]$ \\
\hline
\end{tabular}

\section{Design of Wavelets from Maximally Flat Filter Banks}

The popularity of wavelet analysis comes mainly from its ability to locate and characterize different types of singularities (see [18] for a review). In such applications, nonsubsampled filter banks are not only convenient for implementation in discrete time but also give rise to a broad spectrum of wavelets that can be derived from iterated octave band trees. Note that for singularity detection and discrimination, it is important to use wavelets with a given number of vanishing moments and a certain high degree of regularity.

The procedure for wavelet design from a two-channel filter bank $\left\{H_{0}(z), H_{1}(z)\right\}$, pioneered by Daubechies [2], is basically described by (31) and (34). The regularity of the derived wavelet is closely related to the multiplicity of zeros of the lowpass filter $H_{0}(z)$ at $z=-1$. Note that $H_{0}(z)$ has to have a zero at $z=-1$ for the iterative scheme (31) to converge and that highly regular wavelets are obtained from filters with a maximum number of zeros at $z=-1$. On the other hand, the number of vanishing moments of the wavelet is determined by the multiplicity of zeros of the highpass filter $H_{1}(z)$ at $z=1$. Hence, the design of highly regular wavelets from iterated filter banks amounts to the design of maximally flat ${ }^{5}$ filters $H_{0}(z)$ and $H_{1}(z)$. In our case, the relevant issues are the flatness of $H_{0}\left(e^{j \omega}\right)$ at $\omega=\pi$ and the flatness of $H_{1}\left(e^{j \omega}\right)$ at $\omega=0$. We study the design under the constraint that $H_{0}(z)$ and $H_{1}(z)$ are power complementary, which corresponds to tight frames in $\ell^{2}(\mathbf{Z})$.

\footnotetext{
${ }^{5}$ The flatness of a filter at some frequency is defined as the multiplicity of the root of the first derivative of its frequency response at that frequency. Maximally flat filters are filters with a frequency response whose first derivative has a maximum number of zeros on the unit circle.
}

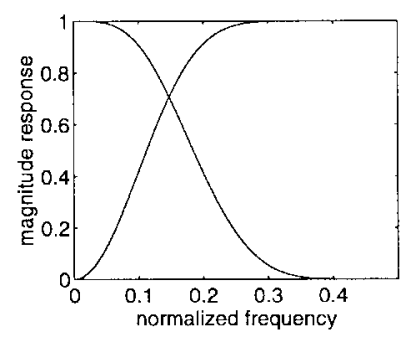

(a)

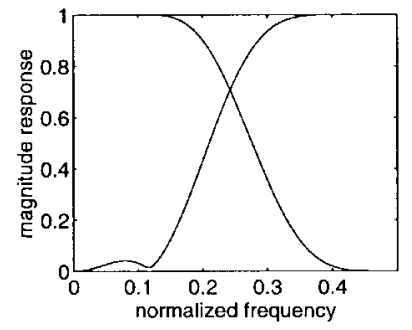

(c)

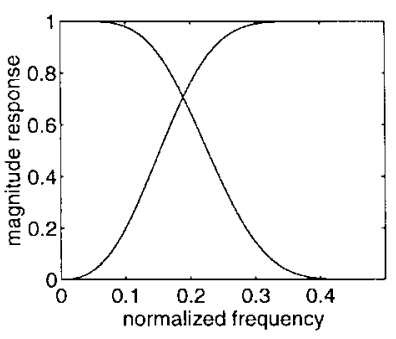

(b)

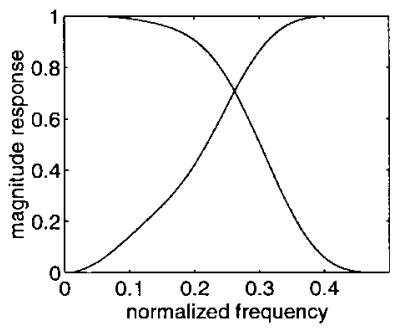

(d)
Fig. 5. Magnitude responses of power complementary filters for generating wavelets with two vanishing moments. The length of the filters is $L=9$, and the highpass filters have a zero of multiplicity $N_{1}=2$ at $z=1$ in all cases. (a) Maximally flat power complementary filters; lowpass filter has $N_{0}=7$ zeros at $z=-1$. (b) Power complementary pair; lowpass filter has $N_{0}=6$ zeros at $z=-1$. (c) Power complementary pair; lowpass filter has $N_{0}=5$ zeros at $z=-1$. (d) Power complementary pair; lowpass filter has $N_{0}=4$ zeros at $z=-1$.

Consider a pair of filters $H_{0}(z)$ and $H_{1}(z)$ satisfying

$$
\begin{aligned}
H_{0}(z) H_{0}\left(z^{-1}\right)+H_{1}(z) H_{1}\left(z^{-1}\right) & =1 \\
H_{0}(-1)=0, \quad H_{1}(1) & =0 .
\end{aligned}
$$

If the multiplicity of the zeros of $H_{0}(z)$ at $z=-1$ is $N_{0}$ and the multiplicity of the zeros of $H_{1}(z)$ at $z=1$ is $N_{1}$, then the filters have flatness $N_{0}-1$ at $\omega=\pi$ and flatness $N_{1}-1$ at $\omega=0$. In designing maximally flat filters, the issue is to maximize $N_{0}+N_{1}$ for a given filter length $L$. This is dealt with in the following proposition.

Proposition 5: For a pair of filters $H_{0}(z)$ and $H_{1}(z)$ of length $L$, satisfying (36) and (37), let $N_{0}$ be the multiplicity of the zeros of $H_{0}(z)$ at $z=-1$, and let $N_{1}$ be the multiplicity of the zeros of $H_{1}(z)$ at $z=1$. It is possible to design $H_{0}(z)$ and $H_{1}(z)$ for any pair of $N_{0}$ and $N_{1}$ such that $1 \leq N_{0}<L, \quad 1 \leq N_{1}<L, \quad N_{0}+N_{1} \leq L$.

A constructive proof of the above proposition is given in [20] as a design procedure for maximally flat power complementary filters. The lowpass filter of the maximally flat pair is obtained as a spectral factor of

$$
H_{0}(z) H_{0}\left(z^{-1}\right)=P\left(\left(\frac{1-z}{2}\right)\left(\frac{1-z^{-1}}{2}\right)\right)
$$

where $P(y)$ is given by

$$
P(y)=(1-y)^{N_{0}}\left(\sum_{l=0}^{L-1-N_{0}}\left(\begin{array}{c}
N_{0}+l-1 \\
l
\end{array}\right) y^{l}\right) .
$$

The highpass filter $H_{1}(z)$ is obtained from the factorization

$$
H_{1}(z) H_{1}\left(z^{-1}\right)=1-P\left(\left(\frac{1-z}{2}\right)\left(\frac{1-z^{-1}}{2}\right)\right) .
$$


TABLE II

Coefficients of Power Complementary Filters Whose Magnitude Responses Are Plotted in Fig. 5. The Highpass Filter $H_{1}(z)$ Has a Zero of Multiplicity 2 at $z=1$ in All Cases. (a) Maximally Flat Filters. (b) Power Complementary Pair; Lowpass Filter $H_{0}(z)$ Has $N_{0}=6$ Zeros at $z=-1$. (c) Power Complementary Pair; Lowpass Filter $H_{0}(z)$ Has $N_{0}=5$ Zeros at $z=-1$. (d) Power Complementary Pair; Lowpass Filter $H_{0}(z)$ Has $N_{0}=4$ Zeros at $z=-1$

\begin{tabular}{rr|rr|rr|rr}
\hline $\mathrm{a}$ & & \multicolumn{2}{|c|}{$\mathrm{b}$} & \multicolumn{2}{c|c}{} & \multicolumn{1}{c}{$\mathrm{c}$} \\
\hline$H_{0}$ & $H_{1}$ & $H_{0}$ & $H_{1}$ & $H_{0}$ & $H_{1}$ & $H_{0}$ \\
\hline 0.37690273 & 0.53402011 & 0.03078682 & 0.22376086 & 0.04095616 & 0.01495479 & 0.18430940 & 0.10430306 \\
-0.65503275 & -0.61696397 & -0.17864344 & -0.53553401 & 0.21295453 & 0.09754126 & 0.53853041 & 0.40558545 \\
0.09170122 & -0.13570812 & 0.38821680 & 0.38072858 & 0.42509906 & 0.26405461 & 0.41251639 & 0.51532830 \\
0.18384469 & 0.06867234 & -0.47681949 & 0.05794996 & 0.37209430 & 0.37342961 & -0.07678751 & 0.13439669 \\
0.05438736 & 0.08732849 & 0.12287851 & -0.14190924 & 0.07046279 & 0.27343750 & -0.10938868 & -0.16366452 \\
-0.02189883 & 0.04577467 & 0.22906289 & -0.04590050 & -0.04396930 & -0.04530460 & 0.00941692 & 0.05310331 \\
-0.02218162 & 0.01415950 & -0.02304830 & 0.03319186 & -0.04396930 & -0.04530460 & 0.00941692 & 0.05310331 \\
-0.00691311 & 0.00251695 & -0.07359995 & 0.02348454 & 0.01192523 & -0.03504126 & -0.01515299 & 0.00659616 \\
-0.00080969 & 0.00020001 & -0.01883383 & 0.00422792 & 0.00745127 & -0.00714229 & 0.00314598 & -0.00907015 \\
\hline
\end{tabular}

Several design examples are given in Table I. Each row of this table contains coefficients of the autocorrelation function of the lowpass filter of the maximally flat power-complementary pair. These filters are designed for several filter lengths $(L)$ and with different multiplicities $\left(N_{1}\right)$ of the zeros of the highpass filter at $z=1$. In the case $N_{1}=1$, which corresponds to wavelets with a single vanishing moment, the lowpass filter $H_{0}(z)$ is the binomial filter of the corresponding length. In that case, the functions $\phi(x)$ generated by iterated filter bank trees in the manner described by (31) are B splines. It follows from the theory of B-splines that the derived wavelets in this case $\left(N_{1}=1\right)$ are at least $L-3$ times continuously differentiable. Wavelets with two vanishing moments are obtained from filters with $N_{1}=2$. In this case, $H_{0}(z)$ can have all but one of its zeros at $z=-1$, which gives wavelets that are at least $L-3-\frac{1}{2} \log _{2} L$ times continuously differentiable [20]. Note that wavelets with one or two vanishing moments generated from orthogonal filter banks cannot have such a high regularity. The reason is that in the orthogonal design, the multiplicity of the zeros of the lowpass filter at $z=-1$ has to be equal to the multiplicity of the zeros of the highpass filter at $z=1$; the requirements for the small number of vanishing moments and high regularity are then contradictory. Note that for even filter lengths $L$ and $N_{1}=L / 2$, maximally flat power complementary design actually gives Daubechies' filters [2].

To achieve a sufficient regularity, the lowpass filter $H_{0}(z)$ does not necessarily have to be maximally flat at $\omega=\pi$. This brings additional freedom, which can be used to meet other design specifications. We illustrate this point with the following design example.

Example 1: Fig. 5 shows the magnitude responses of several power complementary filters for generating wavelets with two vanishing moments. All filters are of length $L=9$ and differ in the multiplicity of the zeros of the lowpass filter at $z=-1$. The maximally flat pair is shown in Fig. 5(a). The design flexibility obtained by relaxing the maximally flat constraint can be used to attain different bandwidths, as illustrated by the three other examples in the same figure. Coefficients of these filters are given in Table II. Fig. 6 gives the wavelets that are obtained from these filters.

\section{CONCLUSION}

Oversampled filter banks were studied in this paper using the theory of frames. Sufficient and necessary conditions on

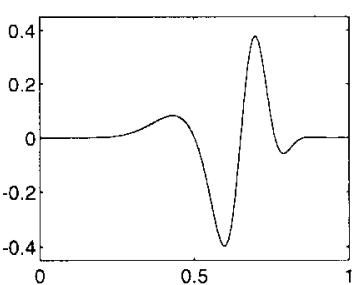

(a)

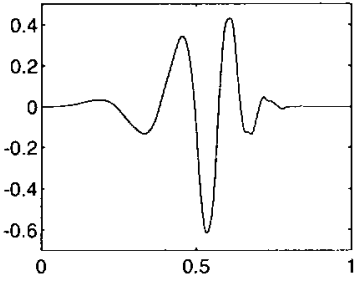

(c)

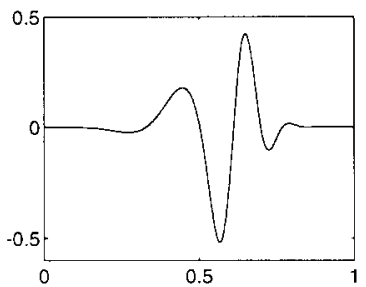

(b)

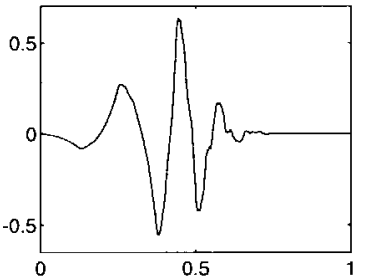

(d)
Fig. 6. Wavelets derived from the filters shown in Fig. 5. Wavelets in (a)-(d) are derived from filters in Fig. 5(a)-(d).

filter banks to implement frame or tight frame expansions in $\ell^{2}(\mathbf{Z})$ were established. In addition, complete parameterizations of FIR filter bank frames, tight frames, and frames for which an FIR synthesis frame exists were given. Nonsubsampled filter banks are studied as a special case. As an illustration of the design flexibility allowed by oversampled filter banks, we described a procedure for design of highly regular wavelets with a given number of vanishing moments.

\section{APPENDIX A}

The proofs given in this Appendix require the introduction of the polyphase representation of a signal $x$ as

$$
X(z)=\sum_{l=0}^{N-1} z^{-l} X_{l}\left(z^{N}\right)
$$

where

$$
X_{l}(z)=\sum_{n=-\infty}^{\infty} x[n N+l] z^{-n} .
$$


The norm of $x$ in terms of its polyphase components is given by

$$
\|x\|^{2}=\frac{1}{2 \pi} \sum_{l=0}^{N-1} \int_{-\pi}^{\pi}\left|X_{l}\left(e^{j \omega}\right)\right|^{2} d \omega .
$$

The subband components of $x$ produced at the output of the filter bank $\left\{H_{0}(z), H_{1}(z), \ldots, H_{K-1}(z)\right\}$ will be denoted by $y_{k}, k=0,1, \ldots, K-1$. They are given in the $Z$-transform domain by

$$
\left[Y_{0}(z) \cdots Y_{K-1}(z)\right]^{T}=\mathbf{H}(z)\left[X_{0}(z) \cdots X_{N-1}(z)\right]^{T}
$$

where $\mathbf{H}(z)$ denotes the polyphase analysis matrix of the filter bank.

Proof of Theorem 1:

\section{a) Necessary Condition:}

Suppose $\mathbf{H}\left(e^{j \omega_{0}}\right) \mathbf{a}=\mathbf{0}$ for some vector $\mathbf{a}$ and some frequency $\omega_{0}$. Consider the sequence of signals $\left\{x_{i}\right\}_{i=1}^{\infty}$ such that the polyphase representation of $x_{i}$ has the form

$\left[X_{0}^{(i)}\left(e^{j \omega}\right) X_{1}^{(i)}\left(e^{j \omega}\right) \cdots X_{N-1}^{(i)}\left(e^{j \omega}\right)\right]^{T}= \begin{cases}p_{i}(\omega) \mathbf{a}, & \omega>0 \\ p_{i}(-\omega) \mathbf{a}, & \omega<0\end{cases}$

where

$$
p_{i}(\omega)= \begin{cases}\sqrt{\frac{2 \pi i}{\|a\|}}, & \omega_{0}-\frac{1}{4 i} \leq \omega \leq \omega_{0}+\frac{1}{4 i} \\ 0, & \text { otherwise. }\end{cases}
$$

Let $\left\{y_{0}^{(i)}, y_{1}^{(i)}, \ldots, y_{K-1}^{(i)}\right\}$ be the subband signals produced by $x_{i}$. Then, for all $x_{i},\left\|x_{i}\right\|^{2}=1$, but $\sum_{k=0}^{K-1}\left\|y_{k}^{(i)}\right\|^{2} \rightarrow 0$ as $i \rightarrow \infty$. Therefore, there is no $A>0$ such that

$$
A\|x\|^{2} \leq \sum_{k=0}^{K-1}\left\|y_{k}\right\|^{2}
$$

for all $x \in \ell^{2}(\mathbf{Z})$.

b) Sufficient Condition:

Consider

$$
\sum_{k=0}^{K-1}\left\|y_{k}\right\|^{2}=\sum_{k=0}^{K-1} \frac{1}{2 \pi} \int_{-\pi}^{\pi}\left|\sum_{l=0}^{N-1} H_{k, l}\left(e^{j \omega}\right) X_{l}\left(e^{j \omega}\right)\right|^{2} d \omega .
$$

Since we are considering FIR filter banks (or stable IIR filters, i.e., no poles on the unit circle)

$$
\begin{aligned}
\sum_{k=0}^{K-1}\left\|y_{k}\right\|^{2} & \leq \sum_{k=0}^{K-1} \frac{1}{2 \pi} \int_{-\pi}^{\pi} \sum_{l=0}^{N-1}\left|H_{k, l}\left(e^{j \omega}\right) X_{l}\left(e^{j \omega}\right)\right|^{2} d \omega \\
& =\sum_{l=0}^{N-1} \frac{1}{2 \pi} \int_{-\pi}^{\pi} \sum_{k=0}^{K-1}\left|H_{k, l}\left(e^{j \omega}\right)\right|^{2}\left|X_{l}\left(e^{j \omega}\right)\right|^{2} d \omega \\
& \leq \sum_{l=0}^{N-1} \frac{1}{2 \pi} \int_{-\pi}^{\pi} K \beta\left|X_{l}\left(e^{j \omega}\right)\right|^{2} d \omega
\end{aligned}
$$

holds, where $\beta=\sup _{k, l} \sup _{\omega}\left|H_{k, l}\left(e^{j \omega}\right)\right|^{2}$. Therefore

$$
\sum_{k=0}^{K-1}\left\|y_{k}\right\|^{2} \leq B\|x\|^{2}, \quad 0<B=K \beta<\infty, \quad \forall x \in \ell^{2}(\mathbf{Z}) .
$$

Suppose further that $\mathbf{H}(z)$ is of full rank everywhere on the unit circle. Then, $\tilde{\mathbf{H}}(z) \mathbf{H}(z)$ is also of full rank on the unit circle, and hence, $\mathbf{H}^{+}(z)=(\tilde{\mathbf{H}}(z) \mathbf{H}(z))^{-1} \tilde{\mathbf{H}}(z)$ is a matrix of rational functions of $z$ that are bounded (no poles) on the unit circle. We have that

$$
\left[X_{0}(z) \cdots X_{N-1}(z)\right]^{T}=\mathbf{H}^{+}(z)\left[Y_{0}(z) \cdots Y_{K-1}(z)\right]^{T} .
$$

Since

$$
\|x\|^{2}=\frac{1}{2 \pi} \sum_{l=0}^{N-1} \int_{-\pi}^{\pi}\left|X_{l}\left(e^{j \omega}\right)\right|^{2} d \omega
$$

using the same argument as in the above, it can be shown that

$$
\|x\|^{2} \leq \alpha \sum_{k=0}^{K-1}\left\|y_{k}\right\|^{2}, \quad 0<\alpha<\infty .
$$

This proves that

$$
\begin{aligned}
A\|x\|^{2} \leq & \sum_{k=0}^{K-1}\left\|y_{k}\right\|^{2} \leq B\|x\|^{2}, \\
& 0<A \leq B<\infty, \quad \forall x \in \ell^{2}(\mathbf{Z}) .
\end{aligned}
$$

\section{APPENDIX B}

Proof of Theorem 2:

a) Sufficient Condition: This is obvious.

b) Necessary Condition: Let $M_{l, m}\left(e^{j \omega}\right)=\left[\tilde{\mathbf{H}}\left(e^{j \omega}\right)\right.$ $\left.\mathbf{H}\left(e^{j \omega}\right)\right]_{l, m}$. First, note that all elements on the diagonal of $\tilde{\mathbf{H}}(z) \mathbf{H}(z)$ have to be equal to a constant. To verify this, consider a signal $x$ that has only one nonzero polyphase component. Then

$$
\|x\|^{2}=\frac{1}{2 \pi} \int_{-\pi}^{\pi}\left|X_{l}\left(e^{j \omega}\right)\right|^{2} d \omega
$$

for some $l, 0 \leq l \leq N-1$, and

$$
\sum_{k=0}^{K-1}\left\|y_{k}\right\|^{2}=\frac{1}{2 \pi} \int_{-\pi}^{\pi} M_{l, l}\left(e^{j \omega}\right)\left|X_{l}\left(e^{j \omega}\right)\right|^{2} d \omega .
$$

Then, for the tight frame condition to hold, it is necessary that $M_{l, l}\left(e^{j \omega}\right)=c, l=0,1, \ldots N-1$.

Now, suppose that for some $l, m, M_{l, m}\left(e^{j \omega}\right) \equiv 0$ does not hold. Consider $x$ such that only $X_{l}(z)$ and $X_{m}(z)$ are nonzero. Then

$$
\begin{aligned}
& \sum_{k=0}^{K-1}\left\|y_{k}\right\|^{2} \\
& =c\|x\|^{2}+\frac{1}{2 \pi} \int_{-\pi}^{\pi} \operatorname{Re}\left(M_{l, m}\left(e^{j \omega}\right) X_{l}^{*}\left(e^{j \omega}\right) X_{m}\left(e^{j \omega}\right) d \omega .\right.
\end{aligned}
$$

From this, it follows that

$$
\begin{array}{r}
\int_{-\pi}^{\pi} \operatorname{Re}\left(M_{l, m}\left(e^{j \omega}\right) X_{l}^{*}\left(e^{j \omega}\right) X_{m}\left(e^{j \omega}\right)\right) d \omega=0 \\
\forall X_{l}^{*}\left(e^{j \omega}\right), X_{m}\left(e^{j \omega}\right)
\end{array}
$$

and therefore $M_{l, m}\left(e^{j \omega}\right) \equiv 0$.

\section{APPENDIX C}

Readers not familiar with the Smith form of polynomial matrices are advised to read the beginning of Section III-B in order to follow this proof. 
Proof of Theorem 3: The sufficiency of the condition is obvious. Here, we prove that it is also a necessary condition. Given the filter bank polyphase analysis matrix $\mathbf{H}(z)$, let $\mathbf{H}(z)=\mathbf{R}(z) \mathbf{D}(z) \mathbf{C}(z)$ be its Smith form decomposition. $\mathbf{R}(z)$ and $\mathbf{C}(z)$ are products of corresponding elementary matrices, and

$$
\mathbf{D}(z)=\left[\begin{array}{c}
\mathrm{D}_{1}(z) \\
\mathbf{0}
\end{array}\right]
$$

where $\mathrm{D}_{1}(z)$ is a diagonal matrix of monic polynomials. Suppose that the frame dual to the frame associated with this filter bank consists of finite length vectors, i.e., that $\mathbf{H}^{+}(z)=$ $(\tilde{\mathbf{H}}(z) \mathbf{H}(z))^{-1} \tilde{\mathbf{H}}(z)$ is a polynomial matrix. Substituting the Smith decomposition in the equation for the pseudoinverse $\mathbf{H}^{+}(z)$, we obtain

$$
\mathbf{H}^{+}(z)=\mathbf{C}(z)^{-1}(\tilde{\mathbf{D}}(z) \tilde{\mathbf{R}}(z) \mathbf{R}(z) \mathbf{D}(z))^{-1} \tilde{\mathbf{D}}(z) \tilde{\mathbf{R}}(z) .
$$

This expression further gives

$$
\mathbf{C}(z) \mathbf{H}^{+}(z) \tilde{\mathbf{R}}(z)^{-1}=\left(\tilde{\mathbf{D}}_{1}(z) \mathbf{R}_{11}(z) \mathbf{D}_{1}(z)\right)^{-1}\left[\tilde{\mathbf{D}}_{1}(z) \quad \mathbf{0}\right]
$$

where $\mathbf{R}_{11}(z)$ denotes the $N \times N$ upper-left corner submatrix of $\tilde{\mathbf{R}}(z) \mathbf{R}(z)$. It follows that

$$
\mathbf{C}(z) \mathbf{H}^{+}(z) \tilde{\mathbf{R}}(z)^{-1}=\left[\mathbf{D}_{1}(z)^{-1} \mathbf{R}_{11}(z)^{-1} \quad \mathbf{0}\right] .
$$

The matrix $\tilde{\mathbf{R}}(z)$ is unimodular, and therefore, its inverse is a polynomial matrix. Consequently, the left-hand side of (48) is also a polynomial matrix. Therefore $\mathbf{D}_{1}(z)^{-1} \mathbf{R}_{11}(z)^{-1}$ is a polynomial matrix. This implies that $\operatorname{det}\left(\mathbf{D}_{1}(z) \mathbf{R}_{11}(z)\right)=$ $c \cdot z^{k}$ for some constant $c$ and an integer $k$. However, this is possible only if polynomials on the diagonal of $\mathbf{D}_{1}(z)$ are monomials.

Taking this fact into account, the expression $\mathbf{H}^{+}(z)=$ $(\tilde{\mathbf{H}}(z) \mathbf{H}(z))^{-1} \tilde{\mathbf{H}}(z)$ can be transformed into

$$
\mathbf{H}^{+}(z) \tilde{\mathbf{R}}(z)^{-1}=(\tilde{\mathbf{H}}(z) \mathbf{H}(z))^{-1}\left[\tilde{\mathbf{C}}(z) \tilde{\mathbf{D}}_{1}(z) \quad 0\right] .
$$

From this, we obtain that

$$
\mathbf{H}^{+}(z) \tilde{\mathbf{R}}(z)^{-1}\left[\begin{array}{c}
\tilde{\mathbf{D}}_{1}(z)^{-1} \tilde{\mathbf{C}}(z)^{-1} \\
\mathbf{0}
\end{array}\right]=(\tilde{\mathbf{H}}(z) \mathbf{H}(z))^{-1} .
$$

Since both $\tilde{\mathbf{R}}(z)$ and $\tilde{\mathbf{C}}(z)$ are unimodular, and $\tilde{\mathbf{D}}_{1}(z)$ is a diagonal matrix of monomials, $\tilde{\mathbf{R}}(z)^{-1}, \tilde{\mathbf{C}}(z)^{-1}$, and $\tilde{\mathbf{D}}_{1}(z)^{-1}$ are polynomial matrices. Hence, $(\tilde{\mathbf{H}}(z) \mathbf{H}(z))^{-1}$ must be a polynomial matrix as well, which proves that $\operatorname{det}(\tilde{\mathbf{H}}(z) \mathbf{H}(z))=p(z)$ has to be a monomial. However, this is only possible if $p(z)=$ const since $p(z)=\tilde{p}(z)$. This proves that $\tilde{\mathbf{H}}(z) \mathbf{H}(z)$ has to be unimodular.

\section{APPENDIX D}

Proof of Proposition 2: It is obvious that for any $\mathbf{H}(z)$ of the form given in (18), $\tilde{\mathbf{H}}(z) \mathbf{H}(z)$ has to be unimodular and positive definite on the unit circle.

Suppose that $\mathbf{P}(z)$ is an $N \times N$ unimodular parahermitian polynomial matrix, positive definite on the unit circle. Any $N \times N$ parahermitian matrix of polynomials $\mathbf{P}(z)$ can be factored as

$$
\mathbf{P}(z)=\tilde{\mathbf{H}}(z) \mathbf{H}(z)
$$

where $\mathbf{H}(z)$ is an $N \times N$ matrix of polynomials [14]. Let $\mathbf{H}(z)=\mathbf{R}(z) \mathbf{D}(z) \mathbf{C}(z)$ be the Smith form decomposition of $\mathbf{H}(z)$. Since $\mathbf{P}(z)$ is unimodular and positive definite on the unit circle, the polynomials on the diagonal of $\mathbf{D}(z)$ have to be nonzero monomials. This proves the proposition.

\section{APPENDIX E}

Proof of Proposition 3: Let the Smith decompositions of the polyphase analysis matrix $\mathbf{H}(z)$ and a corresponding FIR polyphase synthesis matrix $\mathbf{G}(z)$ be

$$
\mathbf{H}(z)=\mathbf{R}_{h}(z)\left[\begin{array}{c}
\mathbf{D}_{h}(z) \\
\mathbf{0}
\end{array}\right] \mathbf{C}_{h}(z)
$$

and

$$
\mathbf{G}(z)=\mathbf{R}_{g}(z)\left[\mathbf{D}_{g}(z) \quad \mathbf{0}\right] \mathbf{C}_{g}(z)
$$

respectively. Here, $\mathbf{D}_{h}(z)$ and $\mathbf{D}_{g}(z)$ are diagonal polynomial matrices. From the condition that $\mathbf{G}(z) \mathbf{H}(z)=\mathbf{I}$ and that $\operatorname{det} \mathbf{R}_{g}(z)=\operatorname{det} \mathbf{C}_{h}(z)=1$, it follows that

$$
\operatorname{det} \mathrm{D}_{g}(z) \cdot \operatorname{det} \mathbf{A}(z) \cdot \operatorname{det} \mathrm{D}_{h}(z)=1
$$

where $\mathbf{A}(z)$ denotes the $N \times N$ submatrix in the upper-left corner of $\mathbf{C}_{g}(z) \mathbf{R}_{h}(z)$. Since $\mathbf{A}(z)$ is a polynomial matrix and both $\mathbf{D}_{h}(z)$ and $\mathbf{D}_{g}(z)$ are diagonal polynomial matrices, (54) can be satisfied only if the polynomials on the diagonal of $\mathrm{D}_{h}(z)$ are monomials.

Conversely, perfect reconstruction can be achieved using the filter bank with the synthesis polyphase matrix $\mathbf{G}(z)=$ $\mathbf{C}_{h}(z)^{-1}\left[\mathbf{D}_{h}(z)^{-1} \mathbf{0}\right] \mathbf{R}_{h}(z)^{-1}$. If in the Smith decomposition of the analysis polyphase matrix $\mathbf{D}_{h}(z)$ is a diagonal matrix of monomials, such a synthesis filter bank is obviously FIR.

\section{ACKNOWLEDGMENT}

The authors are grateful to Dr. T. Kalker and Dr. H. Park for valuable and insightful discussions on this subject. They would also like to thank M. Goodwin and G. Chang for their comments on the paper.

\section{REFERENCES}

[1] D. Gabor, "Theory of communications," J. IEE, vol. 93, no. III, pp. 429-457, 1946

[2] I. Daubechies, Ten Lectures on Wavelets. Philadelphia, PA: SIAM, 1992.

[3] S. Mallat, "A theory of multiresolution signal decomposition: The wavelet transform," IEEE Trans. Pattern Anal. Machine Intell., vol. 11, pp. 674-693, July 1989.

[4] I. Daubechies, "The wavelet transform, time-frequency localization and signal analysis," IEEE Trans. Inform. Theory, vol. 36, pp. 961-1005, Sept. 1990.

[5] P. P. Vaidyanathan, Multirate Systems and Filter Banks. Englewood Cliffs, NJ: Prentice-Hall, 1993.

[6] M. Vetterli and J. Kovačević, Wavelets and Subband Coding. Englewood Cliffs, NJ: Prentice-Hall, 1995.

[7] R. Balian, Un principe d'incertitude fort en théorie du signal on mécanique quantique, C. R. Acad. Sci., Paris, vol. 292, série 2, 1981.

[8] F. Low, "Complete sets of wave packets," in A Passion for Physics-Essays in Honor of Geoffrey Chew. Singapore: World Scientific, 1985, pp. 17-22.

[9] S. Mallat and S. Zhong, "Characterization of signals from multiscale edges," IEEE Trans. Pattern Anal. Mach. Intell., vol. 14, pp. 710-732, July 1992. 
[10] S. Mallat and Z. Zhang, "Matching pursuit with time-frequency dictionaries," IEEE Trans. Signal Processing (Special Issue on Wavelets and Signal Processing), vol. 41, pp. 3397-3415, Dec. 1993.

[11] V. K. Goyal, M. Vetterli, and N. T. Thao, "Efficient representations with quantized matching pursuit," in Proc. 12th Int. Conf. Anal. Opt. Sys.: Images, Wavelets, PDE's, Paris, France, June 1996, pp. 305-311.

[12] R. J. Duffin and A. C. Schaeffer, "A class of nonharmonic fourier series," Trans. Amer. Math. Soc, vol. 72, pp. 341-366, Mar. 1952.

[13] H. J. S. Smith, "On systems of linear indeterminate equations and congruences," Philos. Trans. R. Soc. Lond., vol. 151, pp. 293-326, 1861.

[14] D. C. Youla, "On the factorization of rational matrices," IRE Trans. Inform. Theory, vol. IT-7, pp. 172-189, 1961.

[15] H. Park, T. Kalker, and M. Vetterli, Parahermitian Modules and Lossless Systems, preprint, Univ. Calif., Berkeley, CA, Dec. 1994.

[16] M. V. Wickerhauser, "INRIA lectures on wavelet packet algorithms," Tech. Rep., Dept. Math., Yale Univ., Mar. 1991.

[17] E. P. Simoncelli, W. T. Freeman, E. H. Adelson, and D. J. Hegger, "Shiftable multiscale transforms," IEEE Trans. Inform. Theory, vol. 38, pp. 587-608, Mar. 1992.

[18] S. Mallat and W. L. Hwang, "Singularity detection and processing with wavelets," IEEE Trans. Inform. Theory, vol. 38, pp. 617-643, Mar. 1992.

[19] M. Unser and A. Aldroubi, "General sampling theory for nonideal acquisition devices," NCRR Rep. 16/92, Nat. Inst. Health, Bethesda, MD, 1992.

[20] Z. Cvetković and M. Vetterli, "Discrete-time wavelet extrema representation: Properties and consistent reconstruction," IEEE Trans. Signal Processing, vol. 43, pp. 681-693, Mar. 1995.

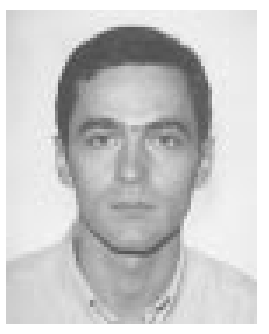

Zoran Cvetković (M'95) received the Dipl. Ing. El. and the Mag. El. degrees from the School of Electrical Engineering, University of Belgrade, Belgrade, Yugoslavia, in 1989 and 1992, respectively, and the Ph.D. degree from the University of California, Berkeley, in 1995.

He worked as a Research and Teaching Assistant at the University of Belgrade, was a Research Assistant at the Center for Telecommunications Research, Columbia University, New York, NY, and was a Research Assistant at the University of California, Berkeley. In the summer of 1994, he worked for Keck Center for Integrative Neuroscience, University of California, San Francisco. In 1996, he was with the Signal Processing Laboratory at EPF Lausanne, Lausanne, Switzerland. Since 1997 he has been with AT\&T Laboratories-Research, Florham Park, NJ. His research interests include time-frequency and time-scale signal analysis, signal processing for telecommunications, audio coding, and modeling of the auditory system.

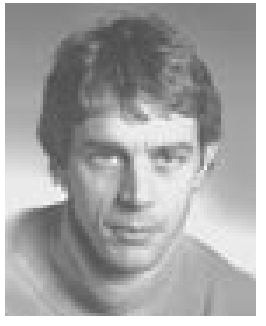

Martin Vetterli (F'95) received the Dipl. El.-Ing. degree from ETH Zürich (ETHZ), Zürich, Switzerland, in 1981, the M.S. degree from Stanford University, Stanford, CA, in 1982, and the Doctorat ès Science degree from EPF Lausanne (EPFL), Lausanne, Switzerland, in 1986.

He was a Research Assistant at Stanford and EPFL and has worked for Siemens and AT\&T Bell Laboratories. In 1986, he joined Columbia University, New York, NY, where he was an Associate Professor of Electrical Engineering and co-director of the Image and Advanced Television Laboratory. In 1993, he joined the University of California, Berkeley, where he is a Professor in the Department of Electrical Engineering and Computer Science. Since 1995, he has been a Professor of Communication Systems at EPFL, and since 1996, he has chaired the Communications Systems Division. His research interests include wavelets, multirate signal processing, computational complexity, signal processing for telecommunications, digital video processing, and compression and wireless video communications.

Dr. Vetterli is a member of SIAM and the Area Editor for Speech, Image, Video, and Signal Processing of the IEEE TRANSACTIONS ON COMMUNICATIONS $\mathrm{He}$ is also on the editorial boards of Signal Processing, Image Communication, Annals of Telecommunications, Applied and Computational Harmonic Analysis, and The Journal of Fourier Analysis and Applications. He received the Best Paper Award of EURASIP in 1984 for his paper on multidimensional subband coding, the Research Prize of the Brown Bovery Corporation (Switzerland) in 1986 for his doctoral thesis, the IEEE Signal Processing Society's 1991 Senior Award (with D. LeGall), the Swiss National Latsis Prize in 1996, and the IEEE Signal Processing Society's 1991 Senior Award (with K. Ramchandran). He was a plenary speaker at the 1992 IEEE ICASSP in San Francisco, CA, and is the co-author, with J. Kovačević, of the book Wavelets and Subband Coding (Englewood Cliffs, NJ: Prentice-Hall, 1995). 